\title{
Incidence Evaluation of SARS-CoV-2 Variants in the Ulsan Area, Korea, Using PowerChek SARS-CoV-2 S-gene Mutation Detection Kit: A Pilot Study
}

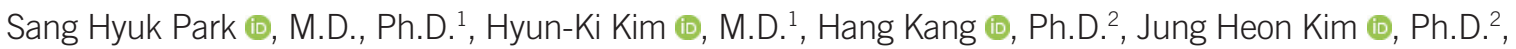 \\ Jaeseung Lee $\mathbb{1}$, M.S. ${ }^{2}$, Ji-Hun Lim $\mathbb{1}$, M.D., Ph.D. ${ }^{1}$, Seon-Ho Lee $\mathbb{0}$, M.D., Ph.D. ${ }^{1}$, and Joseph Jeong $\mathbb{1}$, M.D., Ph.D. ${ }^{1}$ \\ ${ }^{1}$ Department of Laboratory Medicine, University of Ulsan College of Medicine, Ulsan University Hospital, Ulsan, Korea; ${ }^{2}$ Kogene Biotech, Seoul, Korea
}

\section{Dear Editor,}

In Korea, more than 130,000 cases of coronavirus disease 2019 (COVID-19), caused by severe acute respiratory syndrome-coronavirus 2 (SARS-CoV-2), were recorded from January 1, 2020 to May 14, 2021, with approximately 600 cases diagnosed per day following published guidelines for the laboratory diagnosis of COVID-19 in Korea [1]. The number of SARS-CoV-2 variants has been increasing, posing a significant threat in terms of increased infectivity and clinical severity accompanied by reduced immunity upon vaccination [2-4].

Most SARS-CoV-2 variants arise due to changes in the $\mathrm{N}$-terminal domain (NTD) and receptor-binding domain (RBD) [4, 5]. Among NTD variants, the spike deletion H69/N70 (del69-70), which exerts a marginal effect on 50\% neutralization values in plaque reduction neutralization tests of post-vaccination serum samples, is present in the B.1.1.7 variant that was initially dominant in the United Kingdom (UK) before spreading worldwide [6]. N501Y is the most prominent RBD variant, which improves SARS-CoV-2 binding affinity, and is present in the UK (B.1.1.7), the Republic of South Africa (RSA) (B.1.351), and Brazil/Japan (P.1.) variants $[4,6]$. In addition, $\mathrm{K} 417 \mathrm{~N}$, which can improve the receptor binding of SARS-CoV-2, is present in the RSA variant B.1.351, and E484K, associated with immune escape from monoclonal antibodies, has been detected in both the RSA and Brazilian variants $[4,7,8]$.

PowerChek SARS-CoV-2 S-gene Mutation Detection Kit (Kogene Biotech, Seoul, Korea) based on multiplex real-time reverse transcription (rRT)-PCR was developed for detecting COVID-19 variants, especially the UK (B.1.1.7), RSA (B.1.351), Brazil/Japan (P.1), and UK (B.1.525)/Brazil (P.2) variants. Using this kit, we evaluated the incidence of SARS-CoV-2 variants in the Ulsan area of Korea; to our knowledge, this is the first study to evaluate the incidence of SARS-CoV-2 variants in a specific area of Korea. This study was approved by the Institutional Review Board of Ulsan University Hospital, Ulsan, Korea (approval number: 2021-05-025).

Data from 36 SARS-CoV-2-positive respiratory samples, tested using PowerChek SARS-CoV-2 Real-time PCR Kit (Kogene Biotech) at our institution between April 1 and April 30, 2021, were assessed retrospectively. RNA samples were subjected to SARSCoV-2 variant screening using PowerChek SARS-CoV-2 S-gene Mutation Detection Kit following the manufacturer's instructions. Three mutation sites (N501Y, E484K, and K417N) were screened, and the results were classified as (1) UK (B.1.1.7) variant if only N501Y was detected, (2) Brazil/Japan (P.1) variant if both N501Y and E484K were detected, (3) RSA (B1.351) variant if all three
Received: May 23, 2021

Revision received: June 2, 2021

Accepted: November 25, 2021

Corresponding author: Joseph Jeong, M.D., Ph.D.

Department of Laboratory Medicine, University of Ulsan College of Medicine, Ulsan University Hospital, 877 Bangujin-sunhwando-ro, Dong-gu, Ulsan 44033, Korea

Tel: +82-52-250-7273, Fax: +82-52-250-8270

E-mail: joseph@uuh.ulsan.kr 
variants were detected, and (4) UK (B1.525)/Brazil (P.2) variant if only E484K was detected. Direct sequencing of both the NTD (for the 69/70 deletion) and RBD was subsequently performed to confirm the detected variants, using primers designed in-house (for del69-70: forward 5'-CAC ACG TGG TGT TTA CCC T-3', reverse 5'-GTT AGA CTT CTC AGT GGA AGC A-3'; for RBD: forward 5'-GAG GTG ATG AAG TCA GAC AAA TCG-3', reverse 5'CTC TGT ATG GTT GGT AAC CAA CA-3'). Examples of rRT-PCR and direct sequencing results of SARS-CoV-2 variants are presented in Fig. 1, and the results of all 36 samples are summarized in Table 1.

Using rRT-PCR, N501Y, E484K, and K417N were detected in $34(94.4 \%), 1$ (2.8\%), and $0(0.0 \%)$ samples, respectively, and the incidence rates of the B.1.1.7 (UK) and P.1 (Brazil/Japan) variants were calculated as 33/36 (91.7\%) and 1/36 (2.8\%), respectively. Using direct sequencing, del69-70, N501Y $(A \rightarrow T)$,
A

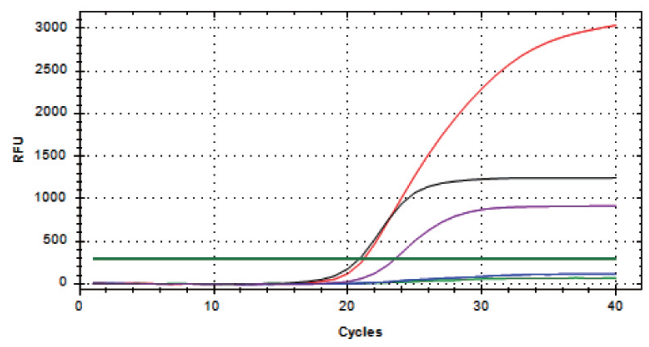

B.1.351 (RSA)

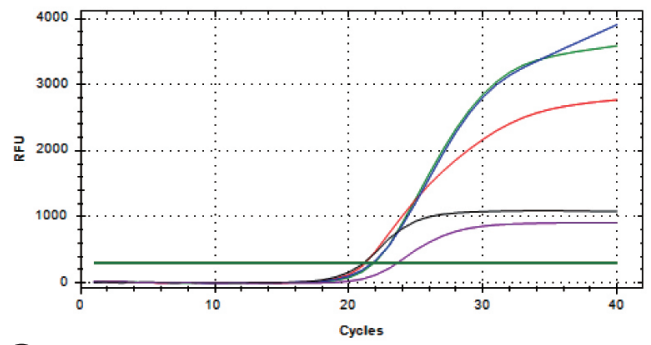

P.1 (Brazil/Japan)

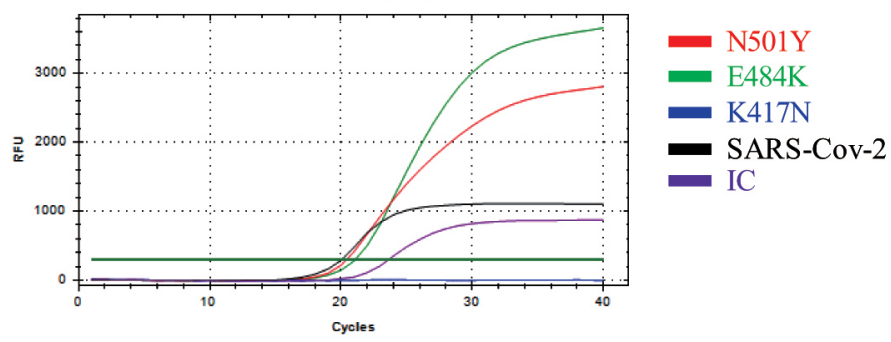

B.1.525 (UK)/P.2 (Brazil)

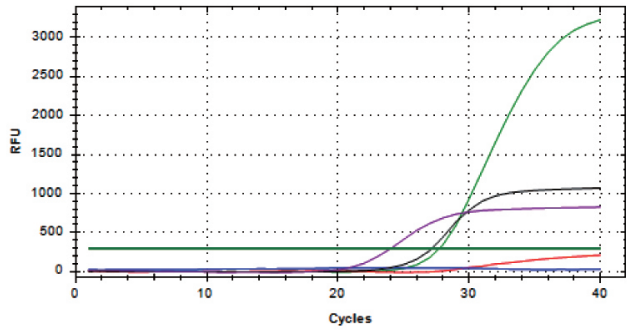

B $69 / 70$ deletion

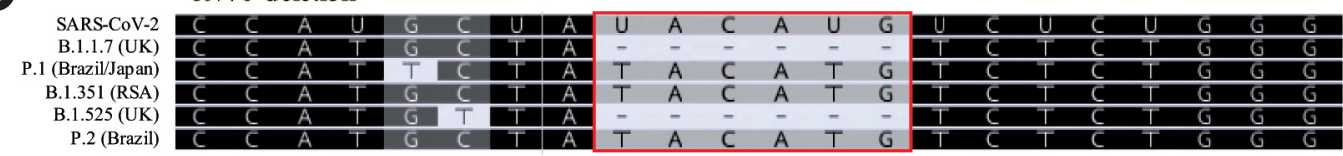

$\mathrm{K} 417 \mathrm{~N}(\mathrm{~T} / \mathrm{G})$

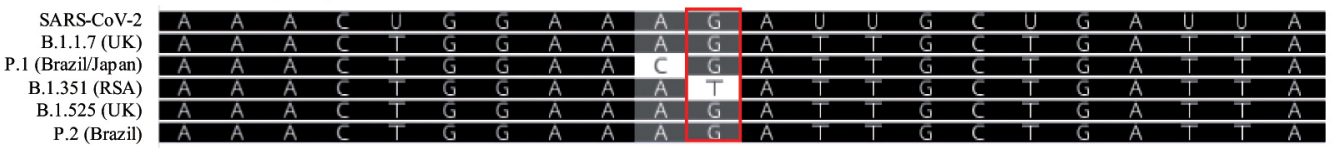

$\mathrm{E} 484 \mathrm{~K}(\mathrm{~A} / \mathrm{G})$

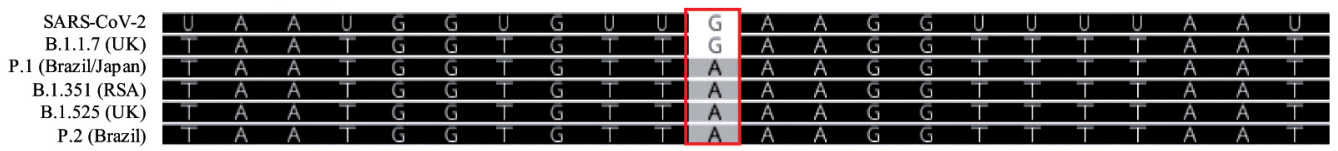

N501Y (T/A)

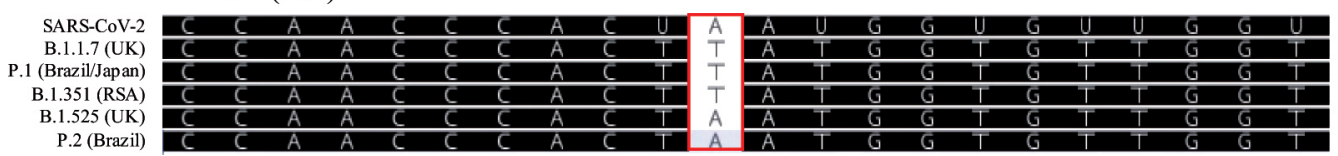

Fig. 1. Examples of rRT-PCR and direct sequencing results of SARS-CoV-2 variants. (A) rRT-PCR results. Amplification curves for N501Y, E484K, K417N, SARS-CoV-2, and the internal control are shown in red, green, blue, black, and purple, respectively. (B) Direct sequencing results for del69-70, K417N, E484K, and N501Y variants.

Abbreviations: rRT-PCR, real-time reverse transcription PCR; N, asparagine; Y, tyrosine; E, glutamate; K, lysine; IC, internal control; UK, United Kingdom; RSA, Republic of South Africa; T, thymine; G, guanine; A, adenine; C, cytosine; U, uracil. 
Park SH, et al.

Incidence of SARS-CoV-2 variants in UIsan, Korea

Table 1. Results of rRT-PCR and direct sequencing of 36 SARS-CoV-2 samples

\begin{tabular}{|c|c|c|c|c|c|c|c|c|c|c|}
\hline \multirow{3}{*}{$\mathrm{N}$} & \multicolumn{5}{|c|}{ rRT-PCR } & \multicolumn{5}{|c|}{ Direct sequencing } \\
\hline & \multirow{2}{*}{$\begin{array}{l}\text { N501Y } \\
\text { (Ct) }\end{array}$} & \multirow{2}{*}{$\begin{array}{c}\text { E484K } \\
\text { (Ct) }\end{array}$} & \multirow{2}{*}{$\begin{array}{l}\text { K417N } \\
\text { (Ct) }\end{array}$} & \multirow{2}{*}{$\begin{array}{c}\text { SARS-CoV-2 } \\
\text { (Ct) }\end{array}$} & \multirow{2}{*}{ Variant } & \multirow{2}{*}{ del69-70 } & \multicolumn{3}{|c|}{ RBD } & \multirow{2}{*}{ Variant } \\
\hline & & & & & & & N501Y (T/A) & E484K (A/G) & K417N (T/G) & \\
\hline 1 & 24.7 & - & - & 22.2 & B.1.1.7 & + & $\mathrm{T}$ & G & G & B.1.1.7 \\
\hline 2 & 27.6 & - & - & 25.7 & B.1.1.7 & + & $\mathrm{T}$ & G & G & B.1.1.7 \\
\hline 3 & 28.3 & - & - & 24.6 & B.1.1.7 & + & $\mathrm{T}$ & G & G & B.1.1.7 \\
\hline 4 & 28.1 & - & - & 25.9 & B.1.1.7 & + & $\mathrm{T}$ & G & G & B.1.1.7 \\
\hline 5 & 33.3 & - & - & 29.4 & B.1.1.7 & + & $\mathrm{T}$ & G & G & B.1.1.7 \\
\hline 6 & 30.2 & - & - & 28.2 & B.1.1.7 & + & $\mathrm{T}$ & G & G & B.1.1.7 \\
\hline 7 & 29.6 & - & - & 27.8 & B.1.1.7 & + & $\mathrm{T}$ & G & G & B.1.1.7 \\
\hline 8 & 31.7 & - & - & 29.3 & B.1.1.7 & + & $\mathrm{T}$ & G & G & B.1.1.7 \\
\hline 9 & 21.7 & - & - & 20.6 & B.1.1.7 & + & $\mathrm{T}$ & G & G & B.1.1.7 \\
\hline 10 & 35.1 & - & - & 30.2 & B.1.1.7 & + & $\mathrm{T}$ & G & G & B.1.1.7 \\
\hline 11 & 35.9 & - & - & 31.0 & B.1.1.7 & + & $\mathrm{T}$ & G & G & B.1.1.7 \\
\hline 12 & 29.9 & - & - & 27.6 & B.1.1.7 & + & $\mathrm{T}$ & G & G & B.1.1.7 \\
\hline 13 & 21.6 & - & - & 20.8 & B.1.1.7 & + & $\mathrm{T}$ & G & G & B.1.1.7 \\
\hline 14 & 20.6 & - & - & 19.8 & B.1.1.7 & + & $\mathrm{T}$ & G & G & B.1.1.7 \\
\hline 15 & 31.6 & 31.9 & - & 29.5 & P.1 & - & $\mathrm{T}$ & A & G & P.1 \\
\hline 16 & - & - & - & 29.4 & SARS-CoV-2 & - & A & G & G & SARS-CoV-2 \\
\hline 17 & 20.0 & - & - & 17.7 & B.1.1.7 & + & $\mathrm{T}$ & G & G & B.1.1.7 \\
\hline 18 & 31.4 & - & - & 29.6 & B.1.1.7 & + & $\mathrm{T}$ & G & G & B.1.1.7 \\
\hline 19 & 25.4 & - & - & 24.3 & B.1.1.7 & + & $\mathrm{T}$ & G & G & B.1.1.7 \\
\hline 20 & 15.6 & - & - & 15.0 & B.1.1.7 & + & $\mathrm{T}$ & G & G & B.1.1.7 \\
\hline 21 & 18.6 & - & - & 18.8 & B.1.1.7 & + & $\mathrm{T}$ & G & G & B.1.1.7 \\
\hline 22 & 12.5 & - & - & 12.1 & B.1.1.7 & + & $\mathrm{T}$ & G & G & B.1.1.7 \\
\hline 23 & 14.9 & - & - & 14.5 & B.1.1.7 & + & $\mathrm{T}$ & G & G & B.1.1.7 \\
\hline 24 & 15.0 & - & - & 14.4 & B.1.1.7 & + & $\mathrm{T}$ & G & G & B.1.1.7 \\
\hline 25 & 31.1 & - & - & 28.8 & B.1.1.7 & + & $\mathrm{T}$ & G & G & B.1.1.7 \\
\hline 26 & 24.7 & - & - & 23.1 & B.1.1.7 & + & $\mathrm{T}$ & G & G & B.1.1.7 \\
\hline 27 & 24.5 & - & - & 22.6 & B.1.1.7 & + & $\mathrm{T}$ & G & G & B.1.1.7 \\
\hline 28 & - & - & - & 25.4 & SARS-CoV-2 & - & A & G & G & SARS-CoV-2 \\
\hline 29 & 28.0 & - & - & 26.4 & B.1.1.7 & + & $\mathrm{T}$ & G & G & B.1.1.7 \\
\hline 30 & 27.3 & - & - & 26.0 & B.1.1.7 & + & $\mathrm{T}$ & G & G & B.1.1.7 \\
\hline 31 & 34.5 & - & - & 32.7 & B.1.1.7 & + & $\mathrm{T}$ & G & G & B.1.1.7 \\
\hline 32 & 24.4 & - & - & 23.0 & B.1.1.7 & + & $\mathrm{T}$ & G & G & B.1.1.7 \\
\hline 33 & 30.8 & - & - & 29.9 & B.1.1.7 & + & $\mathrm{T}$ & G & G & B.1.1.7 \\
\hline 34 & 32.8 & - & - & 30.1 & B.1.1.7 & + & $\mathrm{T}$ & G & G & B.1.1.7 \\
\hline 35 & 33.2 & - & - & 29.4 & B.1.1.7 & + & $\mathrm{T}$ & G & G & B.1.1.7 \\
\hline 36 & 27.2 & - & - & 25.9 & B.1.1.7 & + & $\mathrm{T}$ & G & G & B.1.1.7 \\
\hline
\end{tabular}

-, not determined.

Abbreviations: rRT-PCR, real-time reverse transcription PCR; RBD, receptor-binding domain; N, asparagine; Y, tyrosine; E, glutamate; K, lysine; T, thymine; G, guanine; A, adenine; del, deletion; Ct, cycle threshold. 
E484K $(\mathrm{G} \rightarrow \mathrm{A})$, and $\mathrm{K} 417 \mathrm{~N}(\mathrm{G} \rightarrow \mathrm{T})$ were detected in $33(91.7 \%)$, $34(94.4 \%), 1(2.8 \%)$, and $0(0.0 \%)$ samples, respectively, and the incidence rates of the B.1.1.7 (UK) and P.1 (Brazil/Japan) variants were calculated as 33/36 (91.7\%) and 1/36 (2.8\%), respectively. rRT-PCR and direct sequencing results were concordant in all samples (Table 1).

The incidence rate of the UK variant was very high in the UIsan area. Recent studies have reported that although the UK variant does not hinder vaccine-induced immunity, it may increase fatality by up to $18 \%$ compared with that due to non-UK SARSCoV-2 variants $[9,10]$. These results suggest that UK variant-infected patients need to be managed cautiously. The high incidence of the UK variant in the UIsan area compared with other areas in Korea [11] can be attributed to the frequent entry of foreigners at the Ulsan trading port, increasing the probability of transmission of variants. Although the incidence rates of the RSA and Brazil/Japan variants were low in our study, considering some limitations, including the small sample size, single institutional nature, and use of direct sequencing rather than whole-genome sequencing for validation, a more comprehensive study is needed to confirm these preliminary results.

In conclusion, the incidence of infection due to the UK variant is very high (91.7\%), whereas that due to the RSA and Brazil/ Japan variants is low in the Ulsan area of Korea. A large-scale study is required to confirm our results. Powerchek SARS-CoV-2 S-gene Mutation Detection Kit is useful for the rapid screening of SARS-CoV-2 variants.

\section{ACKNOWLEDGEMENTS}

None.

\section{AUTHOR CONTRIBUTIONS}

Kang $\mathrm{H}$, Kim JH and Lee $\mathrm{J}$ were involved in the implementation of SARS-CoV-2 variant screening. Park SH, Kang H, JH and Lee $\mathrm{J}$ analyzed the data. Park SH drafted the first submitted manuscript and Jeong J supervised the manuscript preparation and submission. Kim HK, Lim JH and Lee SH contributed to the conception. All authors reviewed the manuscript and provided critical feedback.

\section{CONFLICTS OF INTEREST}

Kang $\mathrm{H}$, Kim JH and Lee $\mathrm{J}$ are from the Kogene Biotech and these three authors supported the implementation and analysis of SARS-CoV-2 variant screening performed with the PowerChek SARS-CoV-2 S-gene Mutation Detection Kit (Kogene Biotech).

\section{RESEARCH FUNDING}

None declared.

\section{ORCID}

Sang Hyuk Park

Hyun-Ki Kim

Hang Kang

Jung Heon Kim

Jaeseung Lee

Ji-Hun Lim

Seon-Ho Lee

Joseph Jeong https://orcid.org/0000-0001-7284-6273

https://orcid.org/0000-0002-3299-5298 https://orcid.org/0000-0002-5040-8747 https://orcid.org/0000-0002-9290-6162 https://orcid.org/0000-0003-0620-5237 https://orcid.org/0000-0002-8205-9975 https://orcid.org/0000-0001-8611-0400 https://orcid.org/0000-0001-5980-866X

\section{REFERENCES}

1. Hong KH, Lee SW, Kim TS, Huh HJ, Lee J, Kim SY, et al. Guidelines for Laboratory Diagnosis of Coronavirus Disease 2019 (COVID-19) in Korea. Ann Lab Med 2020;40:351-60.

2. Choudhary S, Sreenivasulu K, Mitra P, Misra S, Sharma P. Role of Genetic Variants and Gene Expression in the Susceptibility and Severity of COVID-19. Ann Lab Med 2021;41:129-38.

3. Hu B, Guo H, Zhou P, Shi Z-L. Characteristics of SARS-CoV-2 and COVID-19. Nat Rev Microbiol 2021;19:141-54.

4. Plante JA, Mitchell BM, Plante KS, Debbink K, Weaver SC, Menachery VD. The variant gambit: COVID-19's next move. Cell Host Microbe 2021; 29:508-15.

5. Tortorici MA, Walls AC, Lang Y, Wang C, Li Z, Koerhuis D, et al. Structural basis for human coronavirus attachment to sialic acid receptors. Nat Struct Mol Biol 2019;26:481-9.

6. Xie X, Liu Y, Liu J, Zhang X, Zou J, Fontes-Garfias CR, et al. Neutralization of SARS-CoV-2 spike 69/70 deletion, E484K, and N501Y variants by BNT162b2 vaccine-elicited sera. Nat Med 2021;27:620-1.

7. Starr TN, Greaney AJ, Addetia A, Hannon WW, Choudhary MC, Dingens AS, et al. Prospective mapping of viral mutations that escape antibodies used to treat COVID-19. Science 2021;371:850-4.

8. Weisblum Y, Schmidt F, Zhang F, DaSilva J, Poston D, Lorenzi JC, et al. Escape from neutralizing antibodies by SARS-CoV-2 spike protein variants. Elife 2020;28:e61312.

9. Zhao S, Lou J, Chong MKC, Cao L, Zheng H, Chen Z, et al. Inferring the association between the risk of COVID-19 case fatality and N501Y substitution in SARS-CoV-2. Viruses 2021;13:638.

10. Conti P, Caraffa A, Gallenga CE, Kritas SK, Frydas I, Younes A, et al. The British variant of the new coronavirus-19 (Sars-Cov-2) should not create a vaccine problem. J Biol Regul Homeost Agents 2021;35:1-4.

11. Kim Y, Kim EJ, Lee SW, Kwon D. Review of the early reports of the epidemiological characteristics of the B.1.1.7 variant of SARS-CoV-2 and its spread worldwide. Osong Public Health Res Perspect 2021;12:13948. 